\title{
INTERMODAL INTERACTION OF INTERNAL SOLITARY WAVES
}

\author{
BY \\ CHIA-SHUN YIH \\ University of Michigan, Ann Arbor, Michigan
}

\begin{abstract}
Interaction of internal solitary waves of different modes in an incompressible fluid with an exponential stratification in density is considered. Two such waves are considered. These waves may be going in the same direction or in opposite directions. The analysis shows that the interaction produces two pairs of waves, whose mode indices are the difference and the sum of those of the original waves, respectively. Each pair consists of a right-going wave and a left-going one, and a part that vanishes eventually everywhere. Higher-order terms affecting interaction are briefly discussed. The interaction of more than two waves can be dealt with pair by pair.
\end{abstract}

1. Introduction. Internal solitary waves in an incompressible fluid stratified in density have been studied previously by many researchers. If there are many such waves in the fluid, and they are all of the same mode and going in the same direction, their interaction is governed by the Korteweg-de Vries equation that has been derived, if not in great detail and clarity, in books and articles that touch upon the subject of internal long waves. A beautiful solution by Gardner, Greene, Kruskal, and Miura [1967] of the KdV equation gives the surprising result that solitary waves (of the same mode if they are internal) going in the same direction regain their identities after interaction, each wave having only a phase shift to recall the experience of its encounter with another wave.

In contrast, the interaction of internal solitary waves of different modes has received scant attention, and this paper is addressed to its treatment. It will be shown that when two such waves interact, two pairs of wave motion are produced, each consisting of two waves of the same mode propagating in opposite directions. The modes of these pairs are in general different (except in one special case) from the modes of the original waves in interaction. In addition, some disturbance which eventually vanishes everywhere is produced. This is a very different situation from that resulting from the interaction of solitary waves of the same mode, and is therefore worthy of note.

Received July 15, 1992.

1991 Mathematics Subject Classification. Primary 76C10. 
2. Analysis. Consider an incompressible medium stratified in density between two horizontal boundaries spaced at distance $h$ apart, and let $x$ and $y$ be Cartesian coordinates, with the origin situated in the lower boundary, and with $y$ measured vertically upward. The wave motion under consideration takes place in the $(x, y)$ plane.

In the following, $t$ denotes time, $u$ and $v$ denote velocity components in the directions of increasing $x$ and $y$, respectively, $p$ denotes the pressure, $\bar{\rho}$ (a function of $y$ only) denotes the density in the undisturbed fluid, $\rho$ denotes the density perturbation, and $g$ denotes the gravitational acceleration. The Euler equations are

$$
\begin{aligned}
& (\bar{\rho}+\rho) \frac{D u}{D t}=-p_{x}, \\
& (\bar{\rho}+\rho) \frac{D v}{D t}=-p_{y}-g(\bar{\rho}+\rho),
\end{aligned}
$$

where

$$
\frac{D}{D t}=\frac{\partial}{\partial t}+u \frac{\partial}{\partial x}+v \frac{\partial}{\partial y} .
$$

The equation of incompressibility is

$$
\frac{D}{D t}(\bar{\rho}+\rho)=0,
$$

which allows the continuity equation to take the usual form

$$
u_{x}+v_{y}=0
$$

and thus allows the velocity components to be expressed as

$$
u=\psi_{y}, \quad v=-\psi_{x},
$$

where $\psi$ denotes the stream function.

Upon elimination of $p$ between (1) and (2), we have

$$
(\bar{\rho}+\rho) \frac{D \xi}{D t}-(\bar{\rho}+\rho)_{y} \frac{D u}{D t}+\rho_{x} \frac{D v}{D t}=-g \rho_{x},
$$

in which

$$
\xi=-\psi_{y y}-\psi_{x x}
$$

is the vorticity. Equation (4) can be written

$$
\frac{D \rho}{D t}=\bar{\rho}_{y} \psi_{x} .
$$

We shall use $h$ as the scale of $x$ and $y$, and consider a density stratification given by

$$
\bar{\rho}=\rho_{0} e^{-\beta y},
$$

in which $y$ and therefore $\beta$ are dimensionless. The scale for $t$ is $(h / \beta g)^{1 / 2}$, the scale for $\psi$ is $\left(\beta g h^{3}\right)^{1 / 2}$, and the scale for $\bar{\rho}$ and $\rho$ is $\rho_{0}$. We shall assume the dimensionless $\rho$ to have the form

$$
\rho=\beta e^{-\beta y} \theta .
$$


The scaling implies that for $u, v$, and any wave velocity the scale is $(\beta g h)^{1 / 2}$. Then (7) and (9) can be written, now in dimensionless terms entirely, as

$$
\begin{gathered}
(1+\beta \theta) \frac{D \xi}{D t}-\left(\beta+\beta \theta_{y}\right) \frac{D u}{D t}+\beta \theta_{x} \frac{D v}{D t}=-\theta_{x}, \\
\theta_{t}+u \theta_{x}+v \theta_{y}=-\psi_{x},
\end{gathered}
$$

where $D / D t$ is given by (3), but now in dimensionless terms, and terms of order $O\left(\beta^{2}\right)$ are neglected. Consider first solitary waves going in the same direction.

Equations (12) and (13) have been solved for one solitary wave of any mode. The solutions for the $m$ th and $n$th modes are, to the lowest order,

$$
\begin{array}{ll}
\psi_{m}=(-1)^{m} a_{m} S_{m}^{2}\left(\sin m \pi y+\frac{\beta}{2} y \sin m \pi y\right), & \theta_{m}=\psi_{m} / c_{m}, \\
\psi_{n}=(-1)^{n} a_{n} S_{n}^{2}\left(\sin n \pi y+\frac{\beta}{2} y \sin n \pi y\right), & \theta_{n}=\psi_{n} / c_{n},
\end{array}
$$

where

$$
\begin{aligned}
S_{m} & =\operatorname{sech} Z_{m}, & & S_{n}=\operatorname{sech} Z_{n}, \\
Z_{m} & =\gamma_{m}\left(x-c_{m} t\right), & & Z_{n}=\gamma_{n}\left(x-c_{n} t\right), \\
c_{m} & =1 /(m \pi), & & c_{n}=1 /(n \pi), \\
\gamma_{m}^{2}=m \pi \beta a_{m} / 9 & \text { for odd } m, & \gamma_{m}^{2} & =m \pi \beta^{2} a_{m} / 36 \text { for even } m,
\end{aligned}
$$

and similarly for $\gamma_{n}^{2}$. We shall need the symbols

$$
T_{m}=\tanh Z_{m}, \quad T_{n}=\tanh Z_{n} .
$$

To fix ideas, we shall assume $n>m$.

For our purpose the term associated with $\beta$ explicitly in (14) or (15) can be dropped for small $\beta$, and we can use

$$
\begin{aligned}
\psi_{m} & =(-1)^{m} a_{m} S_{m}^{2} \sin m \pi y, & \theta_{m} & =\psi_{m} / c_{m}, \\
\psi_{n} & =(-1)^{n} a_{n} S_{n}^{2} \sin n \pi y, & \theta_{n} & =\psi_{n} / c_{n} .
\end{aligned}
$$

We assume

$$
\begin{aligned}
& \psi=\psi_{m}+\psi_{n}+\phi, \\
& \theta=\theta_{m}+\theta_{n}+\theta^{\prime},
\end{aligned}
$$

where $\phi$ and $\theta^{\prime}$ arise from the interaction and are smaller in magnitude than the quantities with subscript $m$ or $n$.

Now we recall that $\psi_{m}$ and $\theta_{m}$ satisfy (12) and (13) by themselves, and so do $\psi_{n}$ and $\theta_{n}$. Hence all terms in these equations containing only the subscript $m$ (or $n$ ), linear or nonlinear, can be removed from them after (23) and (24) have been substituted into them. After this removal, and neglecting higher-order terms, the linear 
terms contain only $\phi$ and $\theta^{\prime}$, and the nonlinear terms are cross products involving subscripts $m$ and $n$. Using (18), we obtain from (12) and (13) the equations

$$
\begin{aligned}
\phi_{y y t}-\theta_{x}^{\prime} & =p q \pi^{2} W, \\
\theta_{t}^{\prime}+\phi_{x} & =p \pi W,
\end{aligned}
$$

where

$$
\begin{gathered}
W=\psi_{n y} \psi_{m x}-\psi_{m y} \psi_{n x}=P \sin p \pi y+Q \sin q \pi y, \\
P=(-1)^{m+n} a_{m} a_{n} \pi\left(n \gamma_{m} R_{1}+m \gamma_{n} R_{2}\right), \\
Q=(-1)^{m+n} a_{m} a_{n} \pi\left(-n \gamma_{m} R_{1}+m \gamma_{n} R_{2}\right), \\
p=n-m, \quad q=n+m, \\
R_{1}=S_{n}^{2} S_{m}^{2} T_{m}, \quad R_{2}=S_{m}^{2} S_{n}^{2} T_{n} .
\end{gathered}
$$

Examination of (25), (26), and (27) shows that we can take

$$
\phi=f_{p} \sin p \pi y+f_{q} \sin q \pi y, \quad \theta^{\prime}=g_{p} \sin p \pi y+g_{q} \sin q \pi y .
$$

We shall treat the $p$-mode only, since the treatment for the $q$-mode is strictly similar. Collecting terms containing $\sin p \pi y$ in (25) and (26), we have

$$
\begin{gathered}
(p \pi)^{2} f_{p t}+g_{p x}=-p q \pi^{2} P, \\
g_{p t}+f_{p x}=p \pi P .
\end{gathered}
$$

Eliminating the function $g_{p}$ between (33) and (34), we have

$$
(p \pi)^{2} f_{p t t}-f_{p x x}=p \pi\left(-q \pi P_{t}-P_{x}\right) .
$$

Similarly

$$
(p \pi)^{2} g_{p t t}-g_{p x x}=p \pi^{2}\left(p^{2} \pi P_{t}+q P_{x}\right) .
$$

Equations (35) and (36) are nonhomogeneous equations of hyperbolic type, and can be solved by integration along the characteristics. We shall solve (35) only, since (36) can be solved similarly. With the new variables

$$
\xi=x-c_{p} t \text { and } \eta=x+c_{p} t,
$$

where $c_{p}=1 /(p \pi),(35)$ becomes

$$
-4 f_{p \xi \eta}=\pi\left[2 m P_{\xi}-2 n P_{\eta}\right] .
$$

Thus

$$
2 f_{p}=\pi \int_{-\infty}^{\eta} m P d \eta-\pi \int_{\infty}^{\xi} n P d \xi
$$

if we integrate from $t=-\infty$ onward. In this $P$ and $Q$ must be expressed in terms of $\xi$ and $\eta$. To do this, we use

$$
\begin{aligned}
x-c_{m} t & =\frac{n}{2 m} \xi+\left(1-\frac{n}{2 m}\right) \eta, \\
x-c_{n} t & =\left(1-\frac{m}{2 n}\right) \xi+\frac{m}{2 n} \eta .
\end{aligned}
$$


Noting the definition of $P$ in (28) and integrating the terms containing $R_{2}$ by parts, we have

$$
4 f_{p}=(-1)^{m+n} a_{m} a_{n} \pi^{2}(\mathrm{I}+\mathrm{II}+\mathrm{III})
$$

where

$$
\begin{aligned}
\mathrm{I} & =n p \gamma_{m} \int_{-\infty}^{\eta} R_{1} d \eta, \\
\mathrm{II} & =-\frac{n^{2} p}{2 n-m} \gamma_{m} \int_{\infty}^{\xi} R_{1} d \xi, \\
\mathrm{III} & =-m\left(n-\frac{n^{2}}{2 n-m}\right) S_{m}^{2} S_{n}^{2} .
\end{aligned}
$$

The integrals in I and II are clearly convergent. Part I will eventually be independent of $\eta$ and represent a wave going in the direction of increasing $x$, and II will eventually be independent of $\xi$ and represent a wave going in the direction of decreasing $x$. Part III will vanish everywhere as $t$ increases, since it is the product of two squares of the sech function with different arguments. Actually we can draw our main conclusion of obtaining two waves with velocities $\pm c_{p}$ from (39). The result (42) is mainly to simplify things a little, and for the convenience of discussing the special case $n=2 m$. The same procedure can be applied to waves of the $q$-mode; we need not write out the results. Thus the offspring of the interacting waves are two pairs of waves, each pair consisting of two oppositely propagating waves of the same mode. The modes of the two pairs are different from each other, and are in general different from the $m$ th and $n$th modes of the interacting solitary waves. This situation is in great contrast with that which prevails in the interaction of solitary waves of the same mode going in the same direction.

But some of the same drama remains. The original waves go on their ways after interaction, retaining their identities. Generally, they do not experience a shift of phase eventually after interaction. But in one special case, that of $n=2 m$, the $m$ wave does experience a shift of phase, so that the drama of the GGKM solution has another echo here. For this case $m=p$, and the $m$-wave is described by functions containing $\xi$ only. This makes the integral in I immediately integrable. The result is

$$
\mathrm{I}=8 m^{2} S_{m}^{2} T_{m} T_{n}
$$

This integral will combine with $\psi_{m}$ to shift its phase without affecting its amplitude.

If we include the terms containing $\beta$ in (14) and (15), the progeny of the interaction will have all the modes. The calculation can be carried out in a strictly similar way. In particular, there will be an $m$-wave and an $n$-wave of this new generation, which will shift the phases of the original $m$-wave and $n$-wave-but only by a much smaller amount than in the special case of $n=2 m$.

Note that if we restrict our attention to the interaction of waves of different modes, we need not require that they all go in one direction. The development given here applies exactly to head-on collisions, upon modifying the signs of $c_{n}$, for instance, if the $n$-wave is going in the direction of decreasing $x$. Furthermore, if there are 
more than two solitary waves interacting with one another, we need simply take them pair by pair, and treat each pair in the same way. We have in this analysis neglected to assign initial phases to the interacting waves, to avoid using too many symbols for little gain in generality. The phases can be put in without affecting the analysis, whenever desirable.

If we include higher-order terms we can investigate in the same way the interaction of two solitary waves of the same mode but different amplitudes. In that case the nonlinear terms would involve waves of all modes. Those arising from either wave alone form an entourage that travels with the same wave. Of the mixed terms, that one having the same mode as the interacting waves plays a role in the $\mathrm{KdV}$ equation, and is taken care of by the GGKM solution. The other modes arising from the mixed terms can be treated in the same way as shown here. These other modes have never been mentioned in the literature, although the elegant GGKM solution of the KdV equation has been cited for dealing with the interaction of internal solitary waves of the same mode and going in the same direction, perhaps because they would clutter up both the analysis and the field of flow, and thus diminish the satisfaction given by the elegant GGKM solution. But they are there, and in compensation they do make the complete picture of solitary-wave interaction more in harmony with our expectation.

\section{REFERENCE}

[1] C. S. Gardner, J. M. Greene, M. D. Kruskal, and R. M. Miura, Method for solving the Korteweg-de Vries equation, Phys. Rev. Lett. 19, 1095-1097 (1967) 